Psychotherapeut 2010 $\cdot 55: 87-88$ DOI 10.1007/s00278-010-0734-y

Online publiziert: 19. Februar 2010

(c) Springer-Verlag 2010

\author{
Manfred Cierpka ${ }^{1}$. Verena Kast ${ }^{2}$ \\ ${ }^{1}$ Institut für Psychosomatische Kooperationsforschung und Familientherapie, \\ Zentrum für Psychosoziale Medizin, Uniklinikum Heidelberg \\ ${ }^{2}$ C.G.-Jung-Institut Zürich, Schweiz
}

\title{
Schwerpunktheft zum Thema Gewalt
}

In der Menschheitsgeschichte ist und war Gewalt stets ein aktuelles Thema. Die 59. Lindauer Psychotherapiewochen befassten sich damit. Ursachen, Erscheinungsformen, Häufigkeiten und Auswirkungen von Gewalt in den unterschiedlichen Facetten, aber auch die Möglichkeiten, der Gewalt zu begegnen, wurden vorgestellt und diskutiert. Das Leitthema der Tagung hieß entsprechend „Der Gewalt begegnen“.

Gewalt hat viele Gesichter: Gewalt geschieht an einzelnen Menschen, etwa im Amoklauf in Winnenden oder in der Schule in Erfurt, aber auch von einem sexuell missbrauchenden Vater an seinem Kind, von einem alkoholisierten, misshandelnden Ehemann an seiner Frau. Gewalt geschieht auch zwischen Gruppen, z. B. Skinheads, die sich mit Autonomen prügeln oder „Ausländer klatschen“. Und Gewalt gibt es schließlich zwischen Stämmen, Völkern oder Nationen z. B. im Mittelalter und im letzten Jahrhundert in Nordirland zwischen Katholiken und Protestanten, bis zum Zweiten Weltkrieg zwischen Deutschen und Franzosen, den Serben und Kroaten im ehemaligen Jugoslawien; aktuell wieder zwischen Hutus und Tutsis in Afrika und in unvorstellbar grausamer Weise im Holocaust von Nationalsozialisten an Juden im Dritten Reich.

Während die schweren kriminellen Delikte von Straftätern über die Jahrzehnte in ihrer Häufigkeit konstant bleiben, beunruhigt die Zunahme der „Beziehungsgewalt“ $z$. B. in Kindergärten, Schulen oder im öffentlichen Raum, die sich mit einer Verrohung der Beziehungskultur in den westlich industrialisierten Ländern in Verbindung bringen lässt. Gleich- zeitig werden inzwischen von vielen Wissenschaftlern und Initiativen Aktionen gegen die Gewalt in all ihren Formen diskutiert und umgesetzt. Es gibt ein breites Spektrum an Präventions- und Interventionsmöglichkeiten.

Im klinisch psychotherapeutischen Kontext begegnet uns die Gewalt in vielen Facetten. Die Opfer, oft Frauen oder Kinder, die durch Gewalthandlungen traumatisiert wurden, benötigen Stabilisierung nach der Tat; manche kommen mit den Symptomen einer posttraumatischen Belastungsstörung Monate oder Jahre später in die Therapie. Wir versuchen in der Traumaarbeit, diesen Patienten wieder Vertrauen in Beziehungen und in die Welt zu vermitteln.

Wir sehen aber auch die Täter, die mit ihren Impulsen und ihrer destruktiven Aggressivität andere Menschen seelisch und körperlich verletzen und sich selbst schaden. Sich den Gefühlen von Hass, Verachtung und Entwertung zu stellen und diese zu bearbeiten, ist eine schwierige, aber notwendige therapeutische Herausforderung.

Der Gewalt entgegenzutreten geht über den psychotherapeutischen Kontext weit hinaus; es ist eine gesellschaftliche Aufgabe und eine Aufgabe des Staates. Die Gesellschaft muss sich dagegen wehren, wenn dissoziales Verhalten Einzelner oder von Gruppen auf Kosten anderer geht und deren Grundrechte verletzt. Freud bezeichnete den Kampf gegen Krieg und Gewalt als Beitrag zur menschlichen Kultur. Eine Überwindung der Gewalt könne sich nur durch Übertragung der Macht an eine größere Einheit ergeben, die durch die Gefühlsbin- dungen der Menschen zusammengehalten wird (Freud 1915). Allerdings scheint diese Kulturleistung nicht von Dauer. Sie bedarf immer neuer Anstrengungen.

Gewaltfreiheit auf Dauer scheint auch eine Illusion zu sein. Das Aufkeimen von Gewalt kann in vielen Kontexten wieder geschehen, auch z. B. auf dem Schulhof, wenn Kinder mit Migrationshintergrund marginalisiert und dann gemobbt werden. Mit Marginalisierung kann es beginnen und mit ethnischen Säuberungen enden, und dem muss man entgegentreten. Die Verantwortung für ein entschlossenes Handeln müssen alle übernehmen.

Im vorliegenden Schwerpunktheft wurden wichtige Referate der Tagung von Autoren zu wissenschaftlichen Beiträgen überarbeitet. Jan Philipp Reemtsma stellt in einer Übersicht die Grundzüge einer Theorie der Gewalt in der Moderne dar und entwickelt ein Konzept des „sozialen Vertrauens“. Annette Streeck-Fischer schildert die Auswirkungen kindlicher Erfahrungen von Misshandlung und Vernachlässigung sowie die Störungen bei Traumatisierungen in der kindlichen Entwicklung, die sich auf die Bindungsfähigkeit auswirken können. Die therapeutische Arbeit fördert - auch mithilfe von Reinszenierungen - die Fähigkeit zu Symbolisierung und Spiel. Den Kampf um Anerkennung bei Gewalt in der Partnerschaft schildert Marga Löwer-Hirsch. Der Wunsch nach Anerkennung durch Andere ist ein legitimes Bedürfnis, das jedoch in seiner übersteigerten oder verleugneten Form eine Gewaltspirale in Gang setzen kann, die in ihren schlimmsten Formen in einen Vernichtungskampf mündet. Hier knüpft Wolfgang Berner in seinem Refe- 
rat über die inneren und äußeren Bedingungen der Beziehung zwischen Sexualität und Gewalt an. Das aktuelle Beispiel der Diskussion um die ständige Zunahme des Angebots von Pornographie im Internet veranschaulicht die Schwierigkeit der Beurteilung und Eingrenzung sexueller Gewalt. Ständig entstehen neue Manifestationen sexueller Gewalt, die neue Formen des therapeutischen Umgangs erforderlich machen.

Carinne Minne stellt schließlich ihre Arbeit im Maßregelvollzug in einer englischen Klinik vor, mit erschütternden Berichten über die psychoanalytische Behandlung zweier Patienten, die getötet haben. Sie beschreibt die Langzeitbehandlungen mithilfe des Strukturmodells der Operationalisierten Psychodynamischen Diagnostik. Auf die erhebliche Angst der die gefährlichen Patienten betreuenden Klinikmitarbeiter wird ausführlich eingegangen, ebenso wie auf den Effekt der „Besserung“ der Patienten.

\section{Manfred Cierpka, Heidelberg}

Verena Kast, St. Gallen, Schweiz

\section{Korrespondenzadresse \\ Prof. Dr. med. Manfred Cierpka}

Institut für Psychosomatische Kooperationsforschung und Familientherapie, Zentrum für Psychosoziale Medizin, Uniklinikum Heidelberg Bergheimer Str. 54, 69115 Heidelberg manfred_cierpka@med.uni-heidelberg.de

\section{Literatur}

Freud S (1915) Zeitgemäßes über Krieg und Tod. Gesammelte Werke, Bd 10. Fischer, Frankfurt a.M., S 323-355

\section{Preise zur Erforschung und Therapie von Persönlichkeits- störungen zu vergeben}

Die Gesellschaft zur Erforschung und Therapie von Persönlichkeitsstörungen (GePs) e.V. und die Asklepios Kliniken Hamburg $\mathrm{GmbH}$ schreiben zum fünften Mal die beiden im deutschen Sprachraum höchstdotierten Preise für klinische Forschung im Bereich der Persönlichkeitsstörungen aus.

Die „Hamburger Fellowship Persönlichkeitsstörungen $2010^{\prime \prime}$ ist mit $5.000 €$ dotiert und richtet sich an Nachwuchswissenschaftlerlnnen. Folgende Richtlinien sind bei der Einreichung zu beachten:

- Alter unter 40 Jahre, nicht habilitiert

- eingereicht werden kann nicht mehr als ein Abstract einer Originalarbeit aus der klinischen Forschung zu Persönlichkeitsstörungen, die noch nicht oder nicht vor 2009 veröffentlicht wurde

- der Einreicher muss Erst- oder Seniorautor sein, die Zustimmung aller Co-Autoren zur Einreichung ist vorzulegen

- die Arbeit soll im deutschen Sprachraum entstanden sein.

Die Fellowship wird zweckgebunden für den Besuch einer internationalen im Bereich der Persönlichkeitsstörungen bedeutenden wissenschaftlichen oder klinischen Einrichtung vergeben. Bewerber werden gebeten, das Abstract ihrer Arbeit in fünf Exemplaren mit einem kurzen CV einzureichen.

Der „Hamburger Preis Persönlichkeitsstörungen 2010" ist mit $10.000 €$ dotiert und wird für Originalarbeiten vergeben, die einen klinischen Bezug aufweisen sollten. Folgende Richtlinien sind bei der Einreichung zu beachten:

- Die Arbeiten sollen noch nicht oder nicht vor 2009 veröffentlicht worden sein - eingereicht werden können nicht mehr als drei Arbeiten (Sonderdrucke oder Manuskripte) von jeweils nicht über 8.000 Worten aus der klinischen Forschung zu Persönlichkeitsstörungen

- der Einreicher muss Erst- oder Seniorautor sein, die Zustimmung aller Co-Autoren zur Einreichung ist vorzulegen

- bei Gruppeneinreichungen soll ein Gruppenvertreter benannt werden
- die Arbeit soll im deutschen Sprachraum entstanden sein.

Das Preisgeld soll für weitere klinische Forschung verwendet werden. Die Arbeiten bzw. Vorschläge sind in fünf Exemplaren mit einem kurzen CV inkl. bisheriger klinischer und wissenschaftlicher Tätigkeit im Bereich der Persönlichkeitsstörungen einzureichen.

Bitte reichen Sie Ihre Abstracts/Arbeiten bis zum 30.04.2010 ein bei:

Dr. Birger Dulz,

Präsident der Gesellschaft zur Erforschung und Therapie von Persönlichkeitsstörungen (GePs) e.V., Asklepios Klinikum Nord, Langenhorner Chaussee 560,

D-22419 Hamburg

Quelle: Gesellschaft zur Erforschung und Therapie von Persönlichkeitsstörungen e.V., www.geps.info 